\title{
Hair Analysis: A Powerful Tool for the Screening of Drugs of Abuse
}

\author{
Christèle Girod* and Christian Staub
}

\begin{abstract}
Nowadays, the analysis of drugs of abuse in hair has become more and more important in most laboratories dealing with forensic toxicological analysis. In fact, hair analysis is considered to be complementary to urine analysis.

In our laboratory, such analyses were first performed in 1995. Since then requests for hair analysis have increased and have become routine.

A method was developed to analyze most drugs of abuse. This method consists of four important steps: Sample preparation, hydrolysis, automatic solid-phase extraction and gas chromatography-mass spectrometry analysis. Particular care has to be taken during sample preparation and in the interpretation of the analytical results. Two other methods were developed, one to confirm consumption of cocaine by the identification of metabolites and to avoid the risk of false-positive results and another to follow subjects under medical heroin treatment.
\end{abstract}

Keywords: Drugs of abuse · Forensics $\cdot$ Hair analysis · Mass spectrometry $\cdot$ Solid-phase extraction

\section{Introduction}

Most toxicological analyses are performed on blood and urine. Since 1979, the analysis for drugs of abuse in hair has been regarded as a tool of interest for toxicologists [1].

Hair grows approximately $1 \mathrm{~cm} /$ month, and drugs held in the hair matrix will follow the growth of the hair shaft. The most significant advantage of hair testing for drugs appears to be its unusually longterm detection window in comparison to other biological samples. Since drugs are deposited in hair, they remain detectable for a period of months to years depending on the hair length. This allows the history of an abuser to be followed by segmentary analysis (Fig. 1).

\footnotetext{
${ }^{*}$ Correspondence: C. Girod

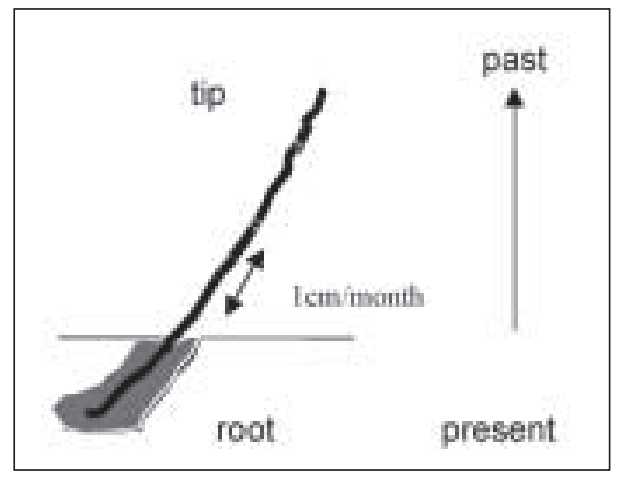

Fig. 1. Segmentary analysis of the hair

In this respect, hair analysis is complementary to urine analysis, which primarily identifies recent drug use, i.e. over the past few days. Moreover, if a positive result is disputed, it is usually possible to collect a second sample from the same growth period and repeat the test.

Actually, different hair analyses have been applied in several different domains: drug treatment programs, prenatal drug exposure, criminal justice, forensic cases. The use of hair analysis in forensic cases depends on the legal situation which differs from country to country. Whereas legislation may be the same for the use of hair analysis in murder cases, the acceptance of hair analytical results varies from region to region concerning drug consumption, drug dealing, general driving ability, and reliability of witnesses and defendants.

This paper demonstrates the importance of hair analysis. The method employed in our laboratory, the problems solved when performing analytical studies and the cut-off values fixed to validate a positive result, will be given.

Other similar methods have been developed to find a marker of illicit heroin use in patients enrolled in a medical heroin treatment or to confirm cocaine consumption by the presence of specific metabolites.

\section{Screening of Drugs for Abuse}

\subsection{Chemical Reagents and Instrumentation}

Methanol, methylene chloride, 2-propanol, hydrochloric acid, phosphate and acetate buffer, and pyridine were supplied by Merck (Darmstadt, Germany). Propionic acid anhydride was provided by Aldrich (Gillingham, U.K.).

6-MAM, codeine, morphine, and nalorphine were obtained from Cambridge Isotope Laboratories Inc.(MA, USA) and Sigma (Buchs, Switzerland), and acetylcodeine from Lipomed (Arlesheim, Switzerland). Hair samples were pulverized 
in a ball mill provided by Retsch (Schieritz, Hauenstein, Switzerland).

Automated extraction was performed on an ASPEC (Gilson Medical Electronics, Villiers-le-Bel, France). Isolute HCX cartridges were provided by IST (Hengoed, U.K.) and used for the extraction.

Hair sample analyses were carried out with a Hewlett Packard (HP) 5890 gas chromatograph equipped with a mass spectrometer HP 5988 operating in electronic impact mode with an energy of $70 \mathrm{eV}$.

\subsection{Sample Preparation}

Soaked hair was prepared according to the procedure described by Edder et al. [2]: $50 \mathrm{mg}$ of blank hair (without drugs) were added to an aqueous solution of drugs. After shaking overnight with a magnetic stirrer, the hair was rinsed with water and methanol to eliminate drugs adsorbed on the surface.

Before analysis, whole hair obtained from a patient was washed successively with $5 \mathrm{ml}$ of methylene chloride, $5 \mathrm{ml}$ of water, and finally $5 \mathrm{ml}$ of methanol. This procedure is very important to eliminate possible external contamination. Finally, the hair was dried for a few min at $60{ }^{\circ} \mathrm{C}$ in a heating block and pulverized $5 \mathrm{~min}$ at $70 \mathrm{~s}^{-1}$ (cycles/second) in a ball mill.

\subsection{Hydrolysis and Extraction of Hair Samples}

Because drugs are fixed inside the hair matrix, a hydrolysis procedure is required before extraction. Ca. $50 \mathrm{mg}$ of powdered soaked hair or real hair sample were placed in a glass tube and $1 \mathrm{ml}$ of $0.01 \mathrm{M}$ hydrochloric acid was added. After incubation at $60^{\circ} \mathrm{C}$ for $12 \mathrm{~h}$, the solution was neutralized with $1 \mathrm{ml}$ of $0.01 \mathrm{M}$ $\mathrm{NaOH}$ and buffered with $1 \mathrm{ml}$ of $1 / 15 \mathrm{M}$ phosphate buffer $\mathrm{pH}$ 7.0. After centrifugation at 4000 r.p.m. for $5 \mathrm{~min}$, the supernatant was transferred into a special glass tube for extraction. The extraction procedure was performed by the ASPEC system which consists of an automated solid-phase extraction.

\subsection{Gas Chromatography-Mass Spectrometry Method}

GC/MS analysis requires a derivatization procedure by propionylation. $100 \mu \mathrm{l}$ of pyridine and $100 \mu \mathrm{l}$ of propionic acid anhydride (PA) were added to the extract and incubated for $30 \mathrm{~min}$ at $60{ }^{\circ} \mathrm{C}$. After evaporation under a nitrogen stream, the extract was reconstituted in $50 \mu \mathrm{l}$ of ethyl acetate and placed in a sample vial for GC/MS analysis.
Helium was used as carrier gas with a capillary column (DB-5MS, $15 \mathrm{~m}$ x $0.25 \mathrm{~mm}$ x $0.25 \mu \mathrm{m}), \mathrm{J} \& \mathrm{~W}$ Scientifics (Folsom, CA). Temperatures: $85^{\circ} \mathrm{C}$ maintained for $1 \mathrm{~min}$ to $190{ }^{\circ} \mathrm{C}$ at $15^{\circ} \mathrm{C} / \mathrm{min}$ and maintained for $0.5 \mathrm{~min}$, to $210^{\circ} \mathrm{C}$ at $2{ }^{\circ} \mathrm{C} / \mathrm{min}$. maintained for $1 \mathrm{~min}$, to $270{ }^{\circ} \mathrm{C}$ at $20^{\circ} \mathrm{C} / \mathrm{min}$. and held at $270{ }^{\circ} \mathrm{C}$ for $8 \mathrm{~min}$. The injector temperature was $270{ }^{\circ} \mathrm{C}$ and injection was made in splitless mode. The source and interface temperatures were $200{ }^{\circ} \mathrm{C}$ and $280{ }^{\circ} \mathrm{C}$, respectively

$3 \mu \mathrm{l}$ of the sample were injected into the GC/MS system which was operating in selected ion monitoring mode (SIM).

Detection was performed with the following ions: codeine $\mathrm{m} / \mathrm{z}=355$ and 282, 6-MAM $\mathrm{m} / \mathrm{z}=383$ and 327, morphine $\mathrm{m} / \mathrm{z}=397$ and 341 , cocaine $\mathrm{m} / \mathrm{z}=303$ and 182 , methadone $\mathrm{m} / \mathrm{z}=294$ and 72, MDMA $\mathrm{m} / \mathrm{z}=114$ and 162 , $\mathrm{MDE} \mathrm{m} / \mathrm{z}=72$ and 162 , and nalorphine $\mathrm{m} / \mathrm{z}=423$ and 367. Quantitation was based on the compound peak area ratios versus the internal standard, nalorphine. The method was validated and the complete procedure has already been described in an earlier article [3]. An example of the chromatogram obtained after the hair extraction of the control sample used in the laboratory is shown Fig. 2 and 3.

\section{Research of Metabolites}

\subsection{Acetylcodeine as a Marker of Illegal Heroin Consumption}

Up to now, heroin exposure was proved by detection of 6-acetylmorphine (6-MAM) (Scheme 1), the major metabo-

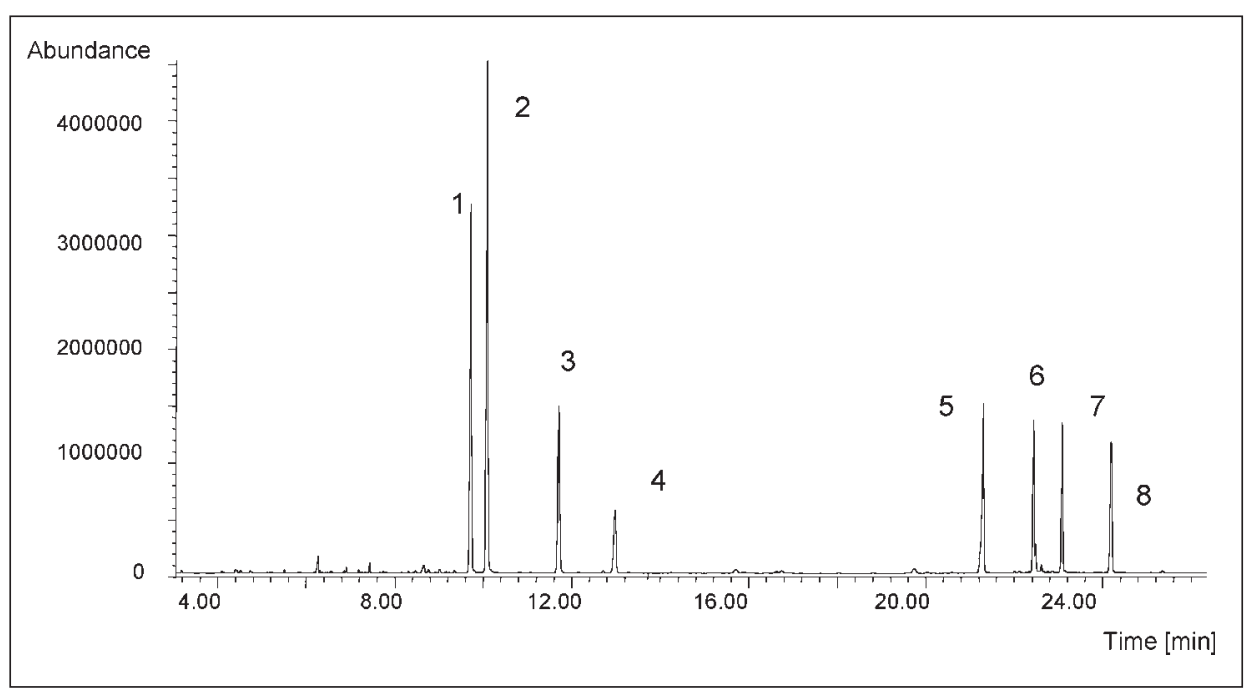

Fig. 2. Limited full-scan GC-MS chromatogram of hair sample control. 1: $M D M A=13 \mathrm{ng} / \mathrm{mg}$; 2: $\mathrm{MDE}=11 \mathrm{ng} / \mathrm{mg} ; 3: \mathrm{MTD}=7 \mathrm{ng} / \mathrm{mg} ; 4: \mathrm{COC}=6 \mathrm{ng} / \mathrm{mg} ; 5: \mathrm{COD}=15 \mathrm{ng} / \mathrm{mg} ; 6: 6-\mathrm{MAM}=$ $12 \mathrm{ng} / \mathrm{mg} ; 7: \mathrm{MOR}=18 \mathrm{ng} / \mathrm{mg} ; 8: \mathrm{NAL}(\mathrm{SI})$. lite found in hair [4]. The difficulty was to find a method which did not transform this compound into morphine.

Heroin maintenance programs have existed for some time and it has been proven that the heroin used for this purpose does not contain acetylcodeine (AC), a manufactured impurity only found in illicit heroin. This is why this compound could become a very interesting marker of illicit heroin use. However, $\mathrm{AC}$ in heroin is reported in a range of $1-15 \%$ only [5]. Therefore, in order to detect $\mathrm{AC}$ at such low concentrations, a sensitive gas chromatography/mass spectrometry method was developed allowing simultaneous quantification of 6-MAM, codeine, morphine, and acetylcodeine in hair.

73 hair samples from heroin abusers and 43 hair samples of medical heroin users were analyzed by the described procedure [6]. In one case of each group the amount of hair sample was not sufficient for analysis, so the patient was not taken into consideration.

AC was detected in $92 \%$ of the illicit heroin samples and only in $12 \%$ of the medical heroin samples.

Generally, the parent drug is the predominant analyte detected in hair samples. However, in this study, about half of the samples have a codeine concentration higher than the corresponding acetylcodeine concentration. This could be explained either by a slight hydrolysis of $\mathrm{AC}$ into $\mathrm{COD}$ or by a simultaneous codeine intake.

In conclusion, even though $\mathrm{AC}$ is found at low concentrations in hair due to its weak presence in illicit heroin, this 


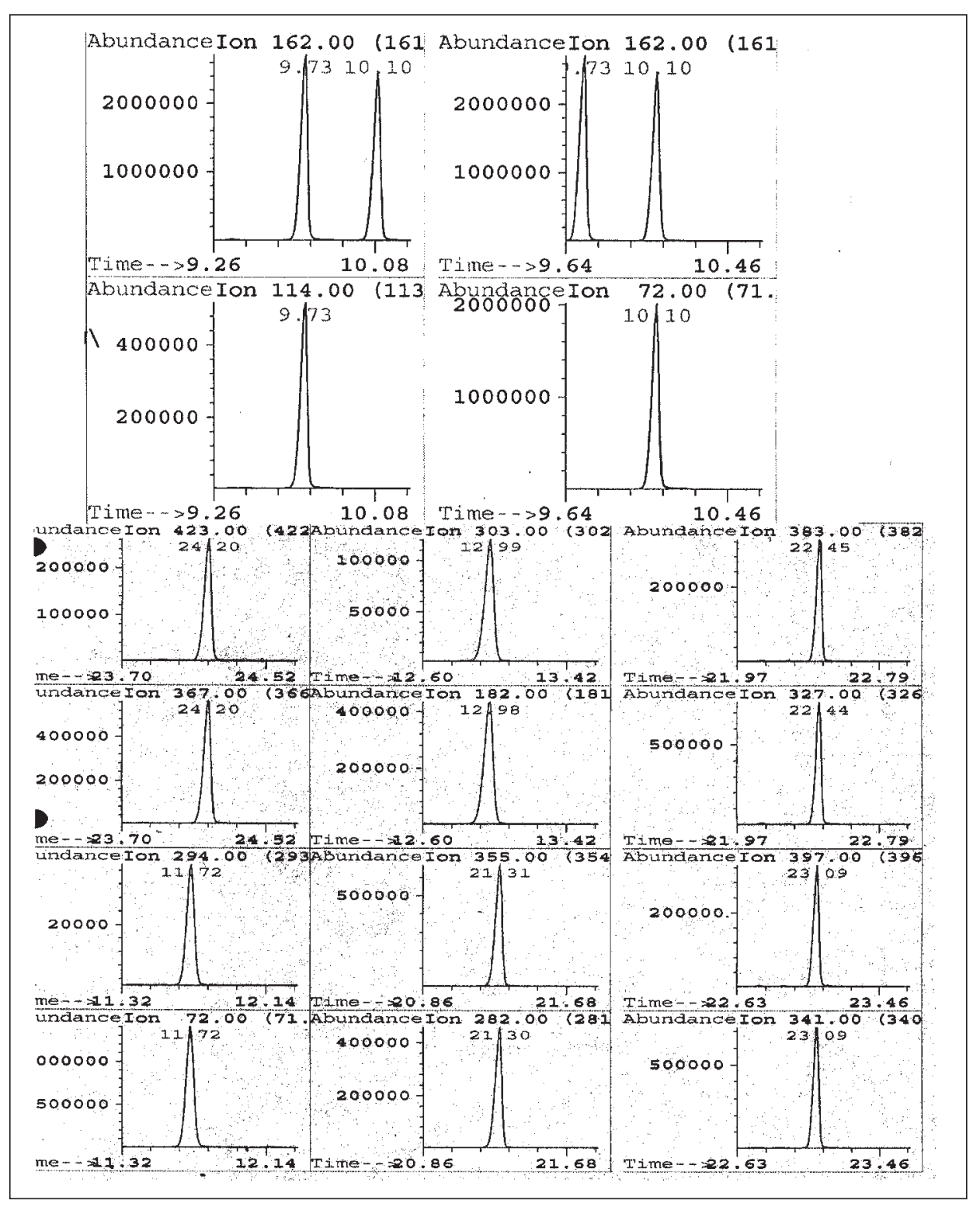

procedure allowed illicit use of heroin to be revealed with a high rate of success $(>90 \%)$.

6-MAM remains certainly the best marker of heroin in hair (rate $=98 \%$ ) but this study has demonstrated that $\mathrm{AC}$ is an interesting biomarker of illicit heroin consumption during the course of a heroin maintenance program.

\subsection{Cocaethylene and Anhydroecgonine Methylester to Prove Cocaine Use}

Cocaine (COC) is an alkaloid found in the plant Erythroxylum Coca which grows in South America. This compound is commonly used and sold on the street. Its principal metabolites are benzoylecgonine (BE) and ecgonine methylester (EME) obtained by chemical and enzymatic hydrolysis respectively [7][8]. Another metabolite, cocaethylene (CET), could be found when COC is consumed with alcohol. This compound is a pharmacologically active analogue of COC but is more potent than COC [9].

Nowadays, COC is often used in the form of the drug called crack. In this case the metabolite obtained by pyrolysis is the anhydroecgonine methylester (AEME) (Scheme 2).

The presence of these metabolites in hair could offer more information of potential benefit in distinguishing drug presence as result of use versus external contamination. They could become suitable markers of cocaine use [10]. Furthermore, CET was present in different human post mortem tissue and this compound proved to be more lethal than $\mathrm{COC}$ [11]. So it could be interesting to identify this compound to interpret data on cocaine-related death [12].

The problem with cocaine is that its major metabolite, BE, can be formed without ingestion of cocaine. That is why
Fig. 3. SIM chromatograph of the hair extract of the control sample it was of interest to develop a method to detect other metabolites and aid in the interpretation of hair samples that have tested positive to cocaine.

Moreover, the aim of developing a specific method was to evaluate the form of cocaine most frequently used by $\mathrm{COC}$ abusers and to determine the prevalence and concentrations of each cocaine metabolite in a sample of cocaine-positive hair.

The method employed the same three steps described previously. Hair sample analyses were carried out with a 3400 gas chromatograph (Varian, USA) equipped with a Ion Trap Saturn 2000 mass spectrometer (Varian, USA) operating in chemical ionization mode with methanol as liquid reagent.

30 hair samples from crack abusers and 30 hair samples of intravenous cocaine users were analyzed by the procedure. The dose of cocaine taken by the 


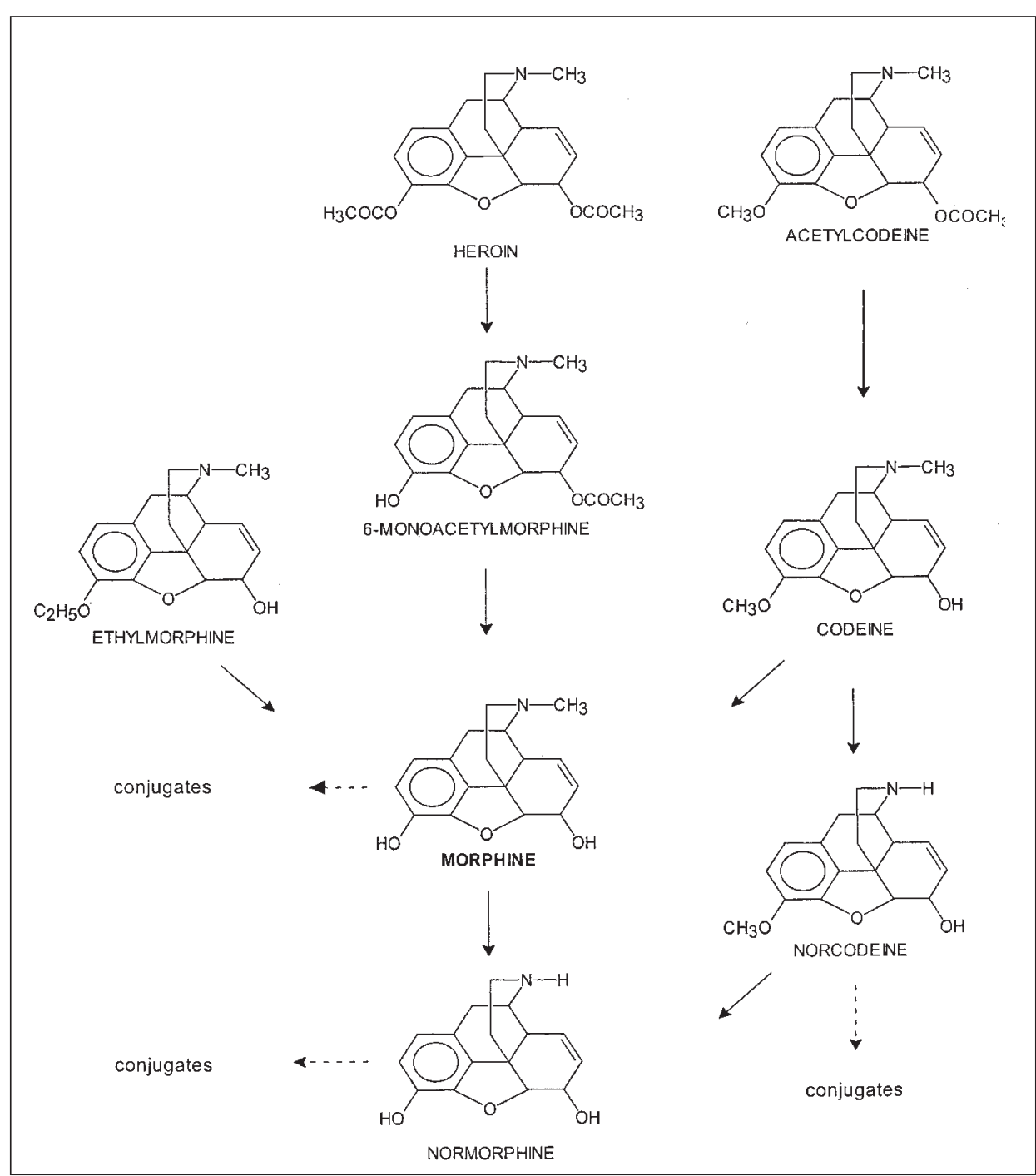

Scheme 1. Metabolic pathway of opiates

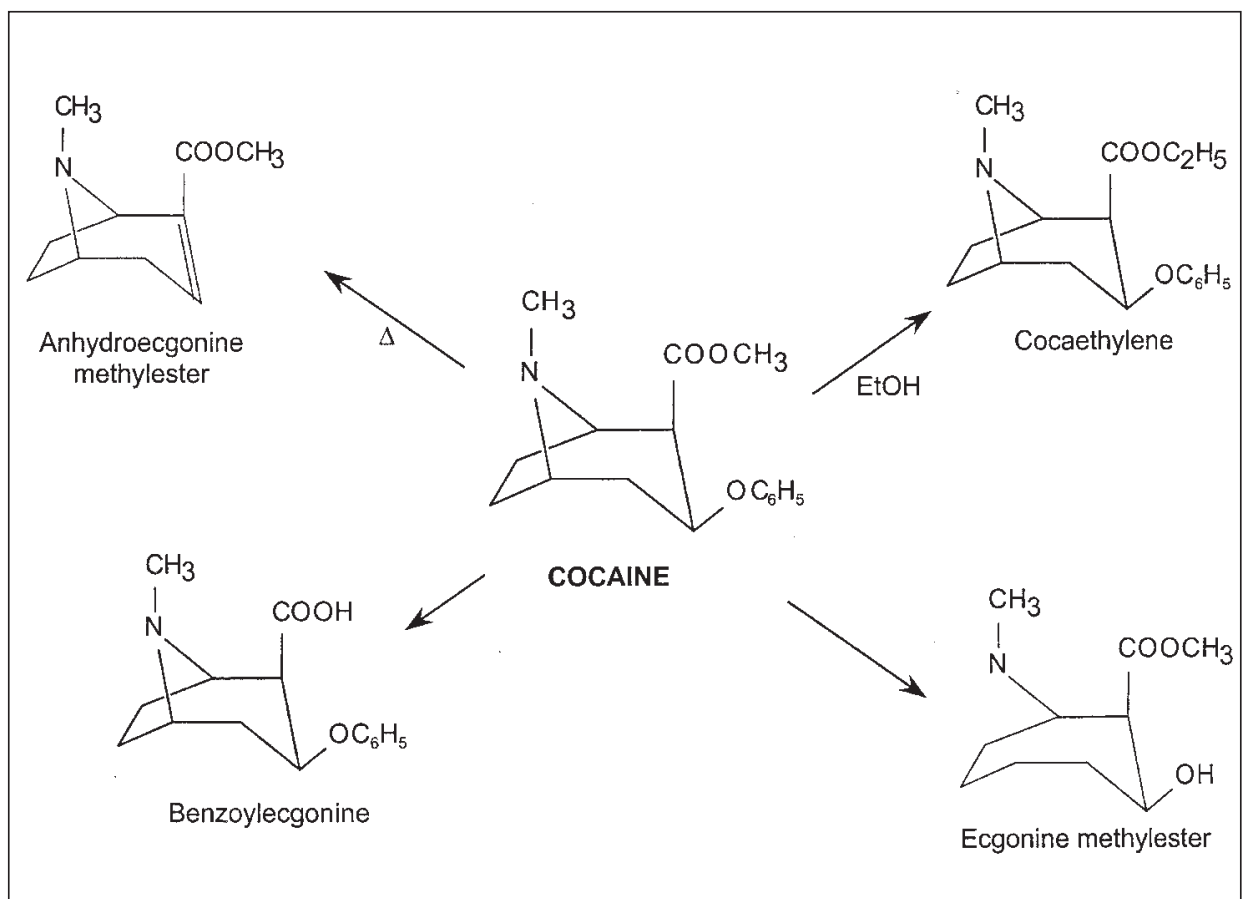

Scheme 2. Metabolic pathway of cocaine abusers was in the same range for the two groups. However, the concentration of cocaine was much higher for the crack abusers than for the intravenous cocaine users. We assume that, as the absorption of the drug is different, the path and fixation in different tissues will not be the same. We observed the presence of AEME in all samples of the first group and in $84 \%$ of the samples of the second group studied. These results showed important concentration values for AEME and indicated that smoking as a means of administration is frequent in Switzerland as opposed to the results found in France by Kintz et al. [10]. These results suggested that AEME could be a suitable marker of crack use. However, care must be taken when low concentrations of cocaine were found because of the formation of an AEME artifact. These results showed also that cocaethylene could be detected in hair and may be a good marker of COC use.

These results are part of a preliminary study and further research has begun to complete and confirm these data.

\section{Limitations of Hair Analysis}

The main problems arose with the questions of external contamination, the routes of incorporation of drugs into hair, possible dose-response relationships, interpretation of results, and their application to forensic and clinical cases.

Many drugs, like cocaine, heroin or marijuana, can be smoked. People in the vicinity of such smokers are exposed to these compounds and the hair analysis will give false-positive results due to passive contamination. The decontamination step is also very important if we consider the possibility of passive exposure. In fact, a universal decontamination method does not exist, each laboratory has its own procedure which means it is difficult to distinguish the limits of true decontamination of the hair and the extraction of the drugs present inside the matrix. So, to differentiate external contamination from consumption, it is necessary to wash the hair shaft. However the search for metabolites is the best approach to confirm drug use.

Another problem, which has often arisen in our laboratory, is the lack of material. The method used was validated for a quantity of $50 \mathrm{mg}$ of hair but sometimes there is not enough hair and axillary or pubic hair has been employed. Therefore, the interpretation of the analytical results cannot be managed in the 
same way. It would be very interesting to develop a more sensitive method where only a small quantity of hair could be used. Moreover, this method could be employed to analyze baby hair to prove eventual drug exposure during the pregnancy.

Nowadays, fashion takes a non-negligible place in the society. More and more people use cosmetic treatment such as bleaching, hair coloration, etc. These transformations affect the hair and by consequence the fixation of the drugs inside the matrix. Thus, determination of the quantity of compounds present in the hair is erroneous. It is important to take this into consideration when the result is near the cut-off (value that is defined in a laboratory and determines the lower limit from which a result is considered positive).

\section{Conclusions}

This paper shows the importance of hair analysis in many forensic applications and the method employed routinely in our laboratory since 1997.

Certainly, many positive results are due to ingestion of drugs. Interpretation of the data must take passive exposure into consideration to determine the source of drugs in any given positive result. It appears that drugs present in sweat can be incorporated into hair. However, the mechanisms of drug accumulation in hair are unknown and there is only limited information on the physicochemical characteristics of how drugs bind to hair. Some evidence suggests that drug incorporation into hair may depend on ethnic hair type, hair color, and hair treatments.

Substantial additional information on the mechanisms of incorporation of drugs into hair, the decontamination of hair, the differentiation between exposure to exogenous and endogenous drugs, and the meaning of the presence of metabolites in the hair are necessary before hair analysis can be used in more forensic applications.

Other studies should be considered if results are to be compared with more personal characteristics, like hair color, melanin content, sex, and age, as these factors could influence drug concentrations in hair and therefore the interpretation. Finally more sensitive methods have to be developed to decrease the quantity of hair necessary for the procedure and to detect weak concentrations of metabolites.

Received: January 29, 2002
[1] A.M. Baumgartner, P.F. Jones, W.A. Baumgartner, C.T. Black, 'Radioimmunoassay of hair for determining opiate-abuse histories', J. Nucl. Med. 1979, 20, 748752.

[2] P. Edder, C. Staub, J.L. Veuthey, I. Pierroz, W. Haerdi, 'Subcritical fluid extraction of opiates in hair of drug addicts', J. Chromatogr, B. Biomed. Appl. 1994, 658, 75-86.

[3] C. Girod, C. Staub, 'Analysis of drugs of abuse in hair by automated solid-phase extraction, GC/EI/MS and GC ion trap/CI/ MS', For. Sci. Int. 2000, 107, 261-271.

[4] B.A. Goldberger, Y.H. Caplan, T. Maguire, E.J. Cone, 'Testing human hair for drugs of abuse. 3. Identification of heroin and 6-acetylmorhine as indicators of heroin use', J. Anal. Toxicol. 1991, 15, 226.

[5] C.L. O'Neal, A. Poklis, 'The detection of acetylcodeine and 6-acetylmorphine in opiate positive urine', For. Sci. Int. 1998, 95, 1-10.

[6] C. Girod, C. Staub, 'Acetylcodeine as a marker of illicit heroin in human hair: Method validation and results of a pilot study', J. Anal. Toxicol. 2001, 25, 106111.

[7] R.C. Baselt, R.H. Cravey, 'Disposition of toxic drugs and chemicals in man', Ed. Year Book Medical Publishers, $3^{\text {rd }}$ Ed., 1989.

[8] 'Clarke's Isolation and Identification of Drugs', The pharmaceutical press, London, 1986.

[9] P. Jatlow, J.D. Elsworth, C.W. Bradberry, G. Winger, J.R. Taylor, R. Russel, R.H. Roth, 'Cocaethylene: a neuropharmacological active metabolite associated with concurrent cocaine-ethanol ingestion', Life Sci. 1991, 48, 1787-1794.

[10] P. Kintz, V. Cirimele, P. Mangin, 'Testing human hair and urine for anhydroecgonine methylester, a pyrolysis product of cocaine', J. Anal. Toxicol. 1995, 19, 479482.

[11] W.L. Hearn, S. Rose, J. Wagner, A. Ciarleglio, D.C. Mash, 'Cocaethylene is more potent than cocaine in mediating lethality', Pharmacol. Biochem. Behav. 1991, 39, 531-533.

[12] A.A. da Matta Chasin, A.F. Midio, 'Validation of an ion-trap gas chromatographic- mass spectrometric method for the determination of cocaine and metabolites and cocaethylene in post mortem whole blood', For. Sci. Int. 2000, 109, 1-13. 